\title{
GLOBAL SOLVABILITY OF AN ABSTRACT COMPLEX
}

\author{
FERNANDO CARDOSO ${ }^{1}$ AND JORGE HOUNIE
}

\begin{abstract}
In a recent paper F. Treves studied a model of complexes of pseudodifferential operators in an open set of $R^{n}$, establishing necessary and sufficient conditions for its semiglobal solvability. In the present paper, the authors give necessary and sufficient conditions for the global solvability of an analogous complex defined on an orientable, compact smooth manifold without boundary.
\end{abstract}

1. Introduction and statement of the theorem. Let $\Omega$ be a $\nu$-dimensional $(\nu \geqslant 1)$, compact, connected, orientable $C^{\infty}$ manifold without boundary. We will denote by $A$ a linear selfadjoint operator, densely defined in a complex Hilbert space $H$, which is unbounded, positive and has a bounded inverse $A^{-1}$. (We may think of $A$ as being, for instance, $\left(1-\Delta_{x}\right)^{s}$ on $\mathbf{R}^{n}$, or a selfadjoint extension of $\left|D_{x}\right|$.) We will use the scale of "Sobolev spaces" $H^{s}$ (for $s \in \mathbf{R}$ ), defined by $A$ : if $s \geqslant 0, H^{s}$ is the space of elements $u$ of $H$ such that $A^{s} u \in H$, equipped with the norm $\|u\|_{s}=\left\|A^{s} u\right\|_{0}$, where \|\|$_{b}$ denotes the norm in $H=H^{0}$; if $s<0, H^{s}$ is the completion of $H$ for the norm $\|u\|_{s}=\left\|A^{s} u\right\|_{0}$. The inner product in $H^{s}$ will be denoted by $(,)_{s}$. Whenever $s \in \mathbf{R}, m \in \mathbf{R}$, $A^{m}$ is an isomorphism (for the Hilbert space structures) of $H^{s}$ onto $H^{s-m}$.

By $H^{\infty}$ we denote the intersection of the spaces $H^{s}$, equipped with the projective limit topology, and by $H^{-\infty}$ their union, with the inductive limit topology. Since for each $s \in \mathbf{R}, H^{s}$ and $H^{-s}$ can be regarded as the dual of each other, so can $H^{\infty}$ and $H^{-\infty}$ : with their topologies, they are the strong dual of each other.

We denote by $C^{\infty}\left(\Omega ; H^{\infty}\right)$ the space of $C^{\infty}$ functions in $\Omega$ valued in $H^{\infty}$. It is the intersection of the spaces $C^{j}\left(\Omega ; H^{k}\right)$ (of the $j$-continuously differentiable functions defined in $\Omega$ and valued in $H^{k}$ ) as the nonnegative integers $j$, $k$, tend to $+\infty$. We equip $C^{\infty}\left(\Omega ; H^{\infty}\right)$ with its natural $C^{\infty}$ topology. We will denote by $\mathscr{D}^{\prime}\left(\Omega ; H^{-\infty}\right)$ the dual of $C^{\infty}\left(\Omega ; H^{\infty}\right)$, and refer to it as the space of distributions in $\Omega$ valued in $H^{-\infty}$. Actually, we shall need forms and currents with values in some topological vector space $E$ (here $E$ will always be either $H^{\infty}$ or $\left.H^{-\infty}\right)$. If $0 \leqslant p \leqslant \nu$, we denote by $\Lambda^{p}{ }^{\prime}(\Omega ; E)$ the space of $E$-valued $p$-currents in $\Omega$ (if $p=0$ they are simply distributions in $\Omega$ valued in $E$ ). In

Received by the editors November 16, 1976.

AMS (MOS) subject classifications (1970). Primary 35N10, 35N15, 35N99, $58 \mathrm{G} 15$.

Key words and phrases. Abstract complexes, global solvability, forms, currents, compatibility conditions, de Rham cohomology.

${ }^{1}$ This work of F. Cardoso was partially supported by CNPq (Brazil). 
terms of local charts $\left(\theta ; t_{1}, \ldots, t_{\nu}\right)$ of $\Omega, \theta \subset \Omega$ an open set, such a current is a linear combination

$$
u=\sum_{|J|=p} u_{J} d t_{J}
$$

where $J$ is an ordered multi-index $\left(j_{1}, \ldots, j_{p}\right)$ of integers such that $1 \leqslant j_{1}$ $<\cdots<j_{p} \leqslant \nu,|J|$ its length, here equal to $p$, and $d t_{J}=d t_{j_{1}} \wedge \cdots \wedge d t_{j_{p}}$. The coefficients $u_{J}$ in (1.1) are $E$-valued distributions in $\theta$, i.e., continuous linear mappings $C_{c}^{\infty}(\theta) \rightarrow E$. Analogously one defines the space $\Lambda^{p} C^{\infty}(\Omega ; E)$ of $E$-valued $C^{\infty} p$-forms in $\Omega$.

Of course, formula (1.1) can be made global by introducing a partition of unity subordinated to a finite covering of $\Omega$ by local charts (in fact, from now on, we shall always assume that such formulas are globally defined in $\Omega$ ). There is a natural coupling between an $E$-valued $p$-current in $\Omega$, such as $u$ in (1.1) and a complex-valued $C^{\infty}(\nu-p)$-form (in $\left.\Omega\right) f=\sum_{|I|=\nu-p} f_{I} d t_{I}$ :

$$
\langle u, f\rangle=\sum_{|J|=p}(-1)^{\sigma} u_{J}\left(f_{J^{*}}\right),
$$

where $J^{*}$ is the (unique) multi-index of length $\nu-p$ such that $J \cap J^{*}=\varnothing$ and $(-1)^{\sigma}$ is the parity of the permutation $J \cup J^{*}$. If both $u$ and $f$ are $C^{\infty}$ forms, we have

$$
\langle u, f\rangle=\int_{\Omega} u \wedge f
$$

If $u \in \Lambda^{p} \Phi^{\prime}(\Omega ; E)$ is given by $(1.1), v=\sum_{\left|J^{\prime}\right|=\nu-p} v_{J^{\prime}} d t_{J^{\prime}}$ is an element of $\Lambda^{\nu-p} C^{\infty}\left(\Omega ; E^{\prime}\right)$, and $\beta$ is the duality pairing between $E$ and $E^{\prime}$, we define

$$
u \wedge_{\beta} v=\sum_{\substack{|J|=p,\left|J^{\prime}\right|=\nu-p}} \beta\left(u_{J}, v_{J^{\prime}}\right) d t_{J} \wedge d t_{J^{\prime}}
$$

The resulting current $u \wedge_{\beta} v$ is an element of $\Lambda^{\nu} \mathscr{D}^{\prime}(\Omega)$, and we may use (1.2) to couple it with the zero-form $\underline{1}$, which is the function identically one in $\Omega$ :

$$
\langle\langle u, v\rangle\rangle=\left\langle u \wedge_{\beta} v, \underline{1}\right\rangle .
$$

Let $d$ stand for the exterior derivative in $\Omega$ (later to be denoted by $d_{t}$, as new "variables" enter in the picture). We recall some facts about the de Rham homology and cohomology with complex coefficients (all forms and currents will have complex values) which will be needed later. Let $\omega \subset \Omega$ be an open set, let $\delta$ denote any one of the symbols $\mathscr{D}^{\prime}, \mathcal{E}^{\prime}, C^{\infty}, C_{c}^{\infty}$ and consider the standard complex:

$$
\begin{aligned}
0 \rightarrow \delta(\omega) \stackrel{d_{0}}{\rightarrow} & \Lambda^{1} \delta(\mu) \rightarrow \cdots \rightarrow \Lambda^{q} \delta(\omega) \stackrel{d_{q}}{\rightarrow} \Lambda^{q+1} \delta(\omega) \\
& \rightarrow \cdots \rightarrow \Lambda^{\nu} \delta(\omega) \stackrel{d_{p}}{\longrightarrow} 0
\end{aligned}
$$

where $d_{q}(q \geqslant 0)$ is the exterior derivative. By definition, the $q$ th de Rham 
cohomology space in $\omega$, which we denote by $\mathfrak{h}^{q}(\omega)$, is the quotient space $\operatorname{Ker} d_{q} / \operatorname{Im} d_{q-1}$, where we take $\delta=C^{\infty}$.

Next we take $\delta=C_{c}^{\infty}$ and we define the $p$ th de Rham homology group in $\omega$ as $\mathfrak{h}_{p}(\omega)=\operatorname{Ker} d_{\nu-p} / \operatorname{Im} d_{\nu-p-1}$. De Rham's theorem states that $\mathfrak{h}^{q}(\omega)$ (resp. $\mathfrak{h}_{p}(\omega)$ ) would have been the same had we taken $\delta$ to be $\mathscr{D}^{\prime}$ (resp. $\mathcal{E}^{\prime}$ : compactly supported currents). For more details on this the reader is referred to [1].

Our basic datum is a $C^{\infty}$ one-form in $\Omega$, depending on the parameter $\lambda \in \sigma(A)$ (the spectrum of $A$ ):

$$
b(t, \lambda)=\sum_{j=1}^{\nu} b_{j}(t, \lambda) d t_{j}
$$

We shall assume that the "convolution operators" $b_{j}(t, A), j=1, \ldots, \nu$, belong to the ring $2_{A}(\Omega)$ defined as follows: the elements of $\mathscr{2}_{A}(\Omega)$ are the series in the nonnegative powers of $A^{-1}$, with complex coefficients in $C^{\infty}(\Omega)$, which converge in $\mathcal{L}(H, H)$ (the Banach space of bounded linear operators on $H$ ), as well as each one of their $t$-derivatives, uniformly with respect to $t$ on $\Omega$.

We shall then form the "differential operator" in $\Omega$

$$
\mathbf{D}=d_{t}+b(t, A) A \wedge .
$$

For each $p=0, \ldots, \nu-1$, it defines a linear operator

$$
\mathbf{D}^{p}: \Lambda^{p} \mathscr{D}^{\prime}(\Omega ; E) \rightarrow \Lambda^{p+1} \mathscr{D}^{\prime}(\Omega ; E) .
$$

We set $\mathbf{D}^{\nu}=0$.

Our first requirement is that (1.8) form a complex, i.e. $\operatorname{Im} \mathbf{D}^{p} \subset \operatorname{Ker} \mathbf{D}^{p+1}$, which means that the standard "Frobenius integrability conditions" are satisfied, and this is well known to be equivalent with the requirement that the 1-form $b(t, \lambda)$ be closed for a.e. $\lambda \in \sigma(A)$.

We shall assume more:

$$
\text { for a.e. } \lambda \in \sigma(A) \text {, the one-form } b(t, \lambda) \text { is exact (in } \Omega) \text {, }
$$

where a.e. is to be understood in the sense of the spectral measure of $A$.

We then introduce a primitive of $b$ :

$$
B(t, \lambda)=\oint_{a}^{t} b(s, \lambda) .
$$

Our purpose is to study the equation

$$
\mathrm{D} u=f,
$$

where $u$ is an $E$-valued $p$-current, $f$ an $H^{\infty}$-valued, $C^{\infty}(p+1)$-form $(p$ $\leqslant \nu-1)$ in $\Omega$. Let us consider a spectral resolution of the operator $A$ (considered in $H$ ):

$$
A=\int \lambda d E(\lambda)
$$


From it one easily derives that a compatibility condition for equation (1.11) is

$$
\text { for a.e. } \lambda \in \sigma(A) \text {, the }(p+1) \text {-form } e^{B(t, A) A} E_{\lambda} f \text { is exact. }
$$

We observe that in case $A$ is a selfadjoint extension of $\left|D_{x}\right|$ on $\mathbf{R}^{n},(1.13)$ corresponds (by Fourier transformation, with respect to $x$ ) to the fact that $d_{t}\left(e^{B(t, \mid \xi)|\xi|} \hat{u}\right)=e^{B(t, \mid \xi)|\xi|} \hat{f}$ for a.e. $\xi$ in $\mathbf{R}_{n}$, i.e.: for a.e. $\xi$ in $\mathbf{R}_{n}$, the $(p+1)-$ form $e^{B(t, \mid \xi)|\xi|} \hat{f}(\xi, t)$ is exact.

The set of forms $f \in \Lambda^{p+1} C^{\infty}\left(\Omega ; H^{\infty}\right)$ satisfying (1.13) will be denoted by $B_{\mathrm{D}}^{p+1} C^{\infty}\left(\Omega ; H^{\infty}\right)$.

It is the goal of the present work to complement (1.13) with conditions bearing on the function $B$ so as to obtain conditions which are necessary as well as sufficient for the "global solvability" of (1.11).

We need the concept of adjoint of the system D. For $u \in \Lambda^{p} \mathscr{D}^{\prime}(\Omega ; E), v$ $\in \Lambda^{\nu-p-1} C^{\infty}\left(\Omega ; E^{\prime}\right)$, we set

$$
\left\langle\left\langle\mathbf{D}^{* p} u, v\right\rangle\right\rangle=(-1)^{p}\left\langle\left\langle u, \mathbf{D}^{\nu-p-1} v\right\rangle\right\rangle .
$$

It is not hard to see that the operators $\mathrm{D}^{* p}(p=0, \ldots, \nu-1)$ are defined by

$$
\mathbf{D}^{*}=-d_{t}+\bar{b}(t, A) A \wedge
$$

where $\bar{b}(t, \lambda)$ is the complex conjugate of the form $b(t, \lambda)$.

We state now our fundamental hypothesis about the primitive $B(t, \lambda)$ or rather about its "principal part".

Let

$$
B(t, \lambda)=B_{0}(t)+B_{1}(t) \lambda^{-1}+\cdots .
$$

For any real number $r$ we write

$$
\Omega_{r}=\left\{t \in \Omega ; \operatorname{Re} B_{0}(t)<r\right\} .
$$

The natural injection of $\Omega_{r}$ into $\Omega$, gives rise to homomorphisms

$$
\mathfrak{h}_{p}\left(\Omega_{r}\right) \stackrel{i_{p}}{\longrightarrow} \mathfrak{h}_{p}(\Omega) .
$$

We also have the transpose homomorphisms (restriction of cohomology classes):

$$
\mathfrak{h}^{p}(\Omega) \stackrel{i_{p}^{*}}{\longrightarrow} \mathfrak{h}^{p}\left(\Omega_{r}\right)
$$

We define the following "global" $(\psi)$ condition (see [2]).

Definition 1.1. We say that the system $\mathbf{D}$ has Property $(\psi)$, in dimension $p$ $(0<p<\nu-1)$, in $\Omega$, if for every real number $r$,

$$
\operatorname{Ker} i_{p}=\{0\}, \text { i.e. } i_{p} \text { is injective, }
$$

or equivalently, 


$$
\operatorname{Im} i_{p}^{*}=\mathfrak{h}^{p}\left(\Omega_{r}\right) \text {, i.e. } i_{p}^{*} \text { is surjective. }
$$

We observe that although Property $(\psi)$ is formulated in terms of the particular primitive (1.10), it depends only on $b$.

We shall state now the main result of this paper:

THEOREM 1.1. The following conditions are equivalent:

(I) The system $\mathbf{D}$ has Property $(\psi)$, in dimension $p$, in $\Omega$.

(II) Given any element $f$ of $\mathfrak{B}_{\mathrm{D}}^{p+1} C^{\infty}\left(\Omega ; H^{\infty}\right)$ there is an element $u$ of $\Lambda^{p} C^{\infty}\left(\Omega ; H^{\infty}\right)$ solution of $\mathrm{D} u=f$ in $\Omega$.

(III) Given any element $f$ of $\mathfrak{B}_{\mathrm{D}}^{p+1} C^{\infty}\left(\Omega ; H^{\infty}\right)$ there is an element $u$ of $\Lambda^{p} \mathscr{D}^{\prime}\left(\Omega ; H^{-\infty}\right)$ solution of $\mathrm{D} u=f$ in $\Omega$.

We thank François Treves for teaching us the results of [2] and for suggesting this problem to us.

2. Proof of Theorem 1.1. It is obvious that (II) $\Rightarrow$ (III).

$(\mathrm{III}) \Rightarrow(\mathrm{I})$. We shall need the following lemmas: the first is a variant of a classical lemma of L. Hörmander (see [2, Lemma II.2.1]).

Lemma 2.1. Suppose that (III) holds. Then there is a constant $C>0$ and an integer $m \geqslant 0$ such that

$$
|\langle\langle f, \bar{v}\rangle\rangle| \leqslant C \sup _{t \in \Omega} \sum_{|\alpha|<m}\left\|\partial_{t}^{\alpha} f\right\|_{m} \sup _{t \in \Omega} \sum_{|\alpha|<m}\left\|\partial_{t}^{\alpha}\left(\mathbf{D}^{*} v\right)\right\|_{m}
$$

whenever $f \in \mathscr{B}_{\mathbb{D}}^{p+1} C^{\infty}\left(\Omega ; H^{\infty}\right), v \in \Lambda^{\nu-p-1} C^{\infty}\left(\Omega ; H^{\infty}\right)$.

The scalar product $\langle\langle\rangle$,$\rangle is defined in (1.5); \|\|_{m}$ is the "Sobolev" norm of order $m$, defined by $A ; \mathbf{D}^{*}$ is the adjoint of $\mathbf{D}$ (see (1.15)).

LEMMA 2.2. Assume that for some real number $r, \mathfrak{h}_{p}\left(\Omega_{r}\right) \rightarrow^{i_{p}} \mathfrak{h}_{p}(\Omega)$ is not injective. Then there exist a real number $r_{0}<r$, a smooth $(\nu-p-1)$-form $v_{0}$ and an exact $(p+1)$-form $f_{0}$ such that

$$
\begin{aligned}
\operatorname{supp} d v_{0} & \subset \Omega_{r_{0}}, \\
\operatorname{supp} f_{0} \cap \Omega_{r_{0}} & =\varnothing, \\
\int_{\Omega} v_{0} \wedge f_{0} & \neq 0 .
\end{aligned}
$$

Proof. According to the hypothesis of the lemma, there is a closed $(\nu-p)$ form $w_{0} \in \Lambda^{\nu-p} C_{c}^{\infty}\left(\Omega_{r}\right)$ which is not compactly exact in $\Omega_{r}$ but it is exact in $\Omega$; hence we may find $v_{0} \in \Lambda^{\nu-p-1} C^{\infty}(\Omega)$ such that $w_{0}=d v_{0}$. Choose $r_{0}<r$ in such a way that the support of $w_{0}$ is contained in $\Omega_{r_{0}}$. By a standard duality argument, there exists a closed $p$-form $g \in \Lambda^{p} C^{\infty}\left(\Omega_{r}\right)$ such that

$$
\int_{\Omega} w_{0} \wedge g \neq 0 .
$$

Let $\zeta \in C_{c}^{\infty}\left(\Omega_{r}\right)$ be equal to one in a neighborhood of $\Omega_{r_{0}}$. Using (2.5), wo obtain 


$$
0 \neq \int_{\Omega} d v_{0} \wedge \zeta g=(-1)^{\nu-p} \int_{\Omega} v_{0} \wedge d(\zeta g)=(-1)^{\nu-p} \int_{\Omega} v_{0} \wedge f_{0}
$$

where $f_{0}=d(\zeta g)$, and this proves the lemma.

We denote by $\chi$ the characteristic function of the open unit interval in $\mathbf{R}$, by $\tau$ a large positive number in $\sigma(A)$. Let $h_{\tau}$ be a unit vector in $H$ such that

$$
E_{\tau+1} h_{\tau}=h_{\tau}, \quad E_{\tau-1} h_{\tau}=0, \quad \tau \in \sigma(A) .
$$

Now we set

$$
\begin{aligned}
& f_{\tau}=\int \chi(\lambda-\tau) e^{-B(t, \lambda) \lambda} d E_{\lambda} h_{\tau} f_{0}=e^{-B(t, A) A} h_{\tau} f_{0}, \\
& v_{\tau}=\int \chi(\lambda-\tau) e^{B(t, \lambda) \lambda} d E_{\lambda} h_{\tau} v_{0}=e^{B(t, A) A} h_{\tau} v_{0}
\end{aligned}
$$

where $f_{0}, v_{0}$ are given by Lemma 2.2 .

Observe that $f_{\tau}, v_{\tau}$ are $C^{\infty}$ forms in $\Omega$, with values in $H^{\infty}$, of degrees $p+1$ and $\nu-p-1$, respectively. Since $f_{0}$ is exact, we derive that

$$
f_{\tau} \in \mathscr{B}_{\mathbf{D}}^{p+1} C^{\infty}\left(\Omega ; H^{\infty}\right) .
$$

Following the arguments of [2], we conclude that inequality (2.1) is not verified for the pair of functions $f_{\tau}, v_{\tau}$, as $\tau \rightarrow+\infty$ in $\sigma(A)$.

(I) $\Rightarrow$ (II). By Whitney's theorem we may assume that $\Omega \subset \mathbf{R}^{2 p+1}$, is an embedding. Let $\tilde{\Omega} \subset \mathbf{R}^{2 v+1}$ be an (open) $\varepsilon$-tubular neighborhood of $\Omega(\varepsilon>0$ small); we may think of $\tilde{\Omega}$ as a "fiber space" over $\Omega$. Let $t^{\prime}=\left(t_{v+1}, \ldots, t_{2 p+1}\right)$ denote the "normal coordinates", in $\tilde{\Omega}$, to $\Omega$. For $\lambda \in \sigma(A)$, we denote by (see (1.6))

$$
\tilde{b}\left(t, t^{\prime}, \lambda\right)=\sum_{j=1}^{\nu} b_{j}(t, \lambda) d t_{j}
$$

the "expanded" $C^{\infty}$ one-form in $\tilde{\Omega}$. We shall form the "expanded differential operator" in $\tilde{\Omega}$,

$$
\tilde{\mathbf{D}}=d_{\left(t, t^{\prime}\right)}+\tilde{b}\left(t, t^{\prime}, A\right) A \wedge
$$

and consider, for each $p=0, \ldots, 2 v+1$, the "expanded" complex (observe that $\tilde{b}\left(t, t^{\prime}, \lambda\right)$ satisfies $\left.(1.9)\right)$ :

$$
\tilde{\mathbf{D}}^{p}: \Lambda^{p} \mathscr{D}^{\prime}(\tilde{\Omega} ; E) \rightarrow \Lambda^{p+1} \mathscr{D}^{\prime}(\tilde{\Omega} ; E), \quad \tilde{\mathbf{D}}^{2 v+1}=0 .
$$

Let

$$
\tilde{B}\left(t, t^{\prime}, \lambda\right)=B(t, \lambda)
$$

with $B(t, \lambda)$ as in (1.10), and write

$$
\tilde{B}\left(t, t^{\prime}, \lambda\right)=B_{0}\left(t, t^{\prime}\right)+B_{1}\left(t, t^{\prime}\right) \lambda^{-1}+\cdots .
$$


For any real number $r$ we set

$$
\tilde{\Omega}_{r}=\left\{\left(t, t^{\prime}\right) \in \tilde{\Omega} ; \operatorname{Re} B_{0}\left(t, t^{\prime}\right)<r\right\} .
$$

We have the commutative diagram:

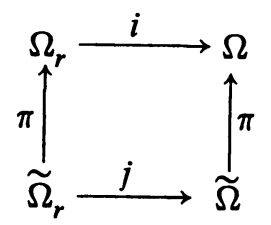

where $i, j$ denote the natural injection and $\pi$ the "fiber projection". This gives rise to a commutative diagram for the induced homomorphisms of the homology groups, where vertical arrows are isomorphisms:

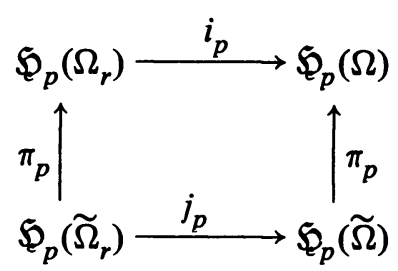

Condition (I) implies that

$$
\operatorname{Ker} j_{p}=\{0\}, \text { i.e. } j_{p} \text { is injective }(0 \leqslant p \leqslant \nu-1) .
$$

Given $f \in \mathscr{B}_{\mathrm{D}}^{p+1} C^{\infty}\left(\Omega ; H^{\infty}\right)$ consider the "expanded" equation

$$
\tilde{\mathbf{D}} \tilde{u}=\tilde{f}
$$

where $\tilde{f}$ is the $H^{\infty}$-valued $C^{\infty}(p+1)$-form in $\tilde{\Omega}$ obtained from $f$ by making it "constant along the fibers". It is easy to verify that

$$
\tilde{f} \in \mathscr{B}_{\tilde{\mathbf{D}}}^{p+1} C^{\infty}\left(\tilde{\Omega} ; H^{\infty}\right) .
$$

Condition (2.18) implies that the relative solvability condition $(\psi)$ (see [2, Definition II.1.1]) is satisfied, in dimension $p$, in $\tilde{\Omega}$, if we take the open set $\theta^{\prime}$ appearing in that definition to be a tubular subneighborhood of $\Omega$. The proof of Theorem II.1.2 of [2] (or rather an abstract version of it) implies that (2.19) can be solved in relatively compact open subsets $\theta^{\prime \prime}$ of $\theta^{\prime}$. Moreover the solution $\tilde{u} \in \Lambda^{p} C^{\infty}\left(\mathcal{\theta}^{\prime \prime} ; H^{\infty}\right)$. If we take $\theta^{\prime \prime} \supset \Omega$ and restrict $\tilde{u}$ to $\Omega$, we obtain a solution of the original equation $\mathrm{D} u=f$, with $u \in \Lambda^{p} C^{\infty}\left(\Omega ; H^{\infty}\right)$. Q.E.D.

REMARK 2.1. A modification of the techniques used in the proof of Theorem II.1.2 in [2], where cubes are replaced by suitable triangulations of $\Omega$, can be applied to give an "intrinsic" proof of (I) $\Rightarrow$ (II).

REMARK 2.2. In case $p=0$, a formula for a solution $u \in C^{\infty}\left(\Omega ; H^{\infty}\right)$ of $\mathbf{D} u=f$ is given by 


$$
u(t)=\oint_{c}^{t} \int_{\sigma(A)} e^{-[B(t, \lambda)-B(s, \lambda)] \lambda} d E_{\lambda} f(s) d s
$$

where $f_{c}^{t}$ indicates a curvilinear integral taken along any path joining $c$ with $t$, $c$ being a point where the real part of $B_{0}(t)$ is minimum. For a discussion of formula (2.21) see [3].

\section{REFERENCES}

1. G. de Rham, Variétés différentiables, Actualités Sci. Indust., no. 1222 = Publ. Inst. Math. Univ. Nancago III, Hermann, Paris, 1955. MR 16, 957.

2. F. Treves, Study of a model in the theory of complexes of pseudodifferential operators, Ann. of Math. 104 (1976), 269-324.

3. Solvability of a model in the theory of complexes of pseudodifferential operators, Lectures given by F. Treves (Recife, 1976), written by J. Hounie (to appear).

Departient of Mathematics, Universidade Fedbral de Pernambuco, Recife, Pe 50 000, Brazn 